\title{
Rotor Angle Position Control with Tracking Error Constraint for Permanent Magnet Synchronous Motors
}

\author{
Daehee Won, Sangjun Lee, Eunchol Shin \\ Robotics R\&D Group, Korea Institute of Industrial Technology \\ 143, Hanggaul-ro, Sangnok-gu, Ansan-si, Gyeonggi-do, Republic of Korea \\ First.daehee@kitech.re.kr; Second.sjlee626@kitech.re.kr; Third.unchol@kitech.re.kr
}

\begin{abstract}
We reviewed the dynamic modeling of a permanent magnet synchronous motor (PMSM) and proposed a rotor angle position control method with an error constraint for the PMSM. To guarantee the tolerance of the position tracking error, a backstepping controller is proposed using the barrier Lyapunov function. In addition, closed-loop stability of the proposed method is analyzed using the Lyapunov theorem.
\end{abstract}

Keywords: PMSM, Barrier Lyapunov function, Backstepping control, Tracking error constraint

\section{Introduction}

Permanent magnet synchronous motors (PMSMs) are receiving increasing attention for electric drive applications such as computer peripherals, robotics, adjustable speed drives and electric vehicles. Based on the characteristics of the model of PMSMs, many modern and intelligent control methods have been applied to PMSMs. These include nonlinear and sliding mode controllers developed to address the speed and position control of PMSMs. Backstepping control is a newly developed technique for the control of uncertain nonlinear systems, particularly those systems that do not satisfy matching conditions [1], and its applications have been proposed [2]. Recently, the barrier Lyapunov function was applied to the backstepping control for output constraints [3] and its applications for various systems have been proposed [4, 5]. This method has the advantage that the control performance of the PMSM can be improved and the control gain can be easily tuned even in the presence of some load fluctuation or system uncertainty. In this study, a position control method with an error constraint is designed for the PMSM. In addition, closed-loop stability of the proposed method is analyzed using the Lyapunov theorem.

\section{Mathematical Modeling}

The mathematical model of a PMSM is described as follows: [6]

$$
\left[\begin{array}{l}
v_{a} \\
v_{b} \\
v_{c}
\end{array}\right]=\left[\begin{array}{ccc}
R_{s} & 0 & 0 \\
0 & R_{s} & 0 \\
0 & 0 & R_{s}
\end{array}\right]\left[\begin{array}{c}
i_{a} \\
i_{b} \\
i_{c}
\end{array}\right]+\left[\begin{array}{ccc}
L_{a} & L_{b a} & L_{c a} \\
L_{b a} & L_{b} & L_{c b} \\
L_{c a} & L_{c b} & L_{c}
\end{array}\right] \frac{d}{d t}\left[\begin{array}{c}
i_{a} \\
i_{b} \\
i_{c}
\end{array}\right]+\left[\begin{array}{c}
e_{a} \\
e_{b} \\
e_{c}
\end{array}\right]
$$

where $v_{a}, v_{b}, v_{c}$ are voltages applied on stator phases $a, b$, and $c$, respectively, $i_{a}, i_{b}, i_{c}$ are phase currents, $e_{a}, e_{b}, e_{c}$ are back EMF voltages, $R_{s}=R_{a}=R_{b}=R_{c}$ are stator phase resistances, $L_{a}, L_{b}, L_{c}$ are phase self inductances and $L_{b a}, L_{c a}, L_{c b}$ are mutual inductances. The back EMF voltage waveforms are expressed by the equation:

$$
\left[\begin{array}{l}
e_{a} \\
e_{b} \\
e_{c}
\end{array}\right]=\omega_{r} \frac{d}{d t}\left[\begin{array}{l}
\Phi_{a} \\
\Phi_{b} \\
\Phi_{c}
\end{array}\right]
$$

where $\Phi_{a}, \Phi_{b}, \Phi_{c}$ are the stator magnetic fluxes of the motor phases $a, b$, and $c$, respectively. As the motor windings are star connected, the next relationship is valid: 


$$
i_{a}+i_{b}+i_{c}=0
$$

Assuming that there is no change in the rotor reluctance with the angular position,

$$
L_{a}=L_{b}=L_{c}=L, \quad L_{b a}=L_{c a}=L_{c b}=M .
$$

Taking into consideration (4), the equation (1) is rearranged as follows:

$$
\left[\begin{array}{l}
v_{a} \\
v_{b} \\
v_{c}
\end{array}\right]=R_{s}\left[\begin{array}{l}
i_{a} \\
i_{b} \\
i_{c}
\end{array}\right]+L_{s} \frac{d}{d t}\left[\begin{array}{c}
i_{a} \\
i_{b} \\
i_{c}
\end{array}\right]+\left[\begin{array}{c}
e_{a} \\
e_{b} \\
e_{c}
\end{array}\right]
$$

where $L_{s}=L-M$ is the stator phase inductance. Hence, the mathematical model of a PMSM is similar to that of a wound rotor synchronous motor. The following assumptions are made in the derivation: 1) Saturation is neglected although it can be taken into account by parameter changes; 2) The back EMF is sinusoidal; 3) Eddy currents and hysteresis losses are negligible, and 4) There are no field current dynamics. With these assumptions the stator $d$, $q$ equations in the rotor reference frame of the PMSM can be expressed as follows:

$$
\begin{aligned}
& v_{d}=R_{s} i_{d}+\frac{d}{d t} \lambda_{d}-\omega_{r} \lambda_{q}, \\
& v_{q}=R_{s} i_{q}+\frac{d}{d t} \lambda_{q}-\omega_{r} \lambda_{d}
\end{aligned}
$$

where $v_{d}$ and $v_{q}$ are the $d$ and $q$ axes components of the voltages of the stator [V], respectively, $i_{d}$ and $i_{q}$ are the $d$ and $q$ axes components of the stator current [A], respectively, $R_{s}$ is the resistance of the stator [W], $\lambda_{d}=L_{d} i_{d}+\lambda_{m}$ and $\lambda_{q}=L_{q} i_{q}$ are $d$ and $q$ axes components of the flux produced by the permanent magnet [Wb], respectively, $\lambda_{m}$ is the magnet mutual flux linkage, and $\omega_{r}$ is the electrical rotor speed [rad/s]. In (6), $v_{d}$ and $v_{q}$ are obtained from voltages applied on stator phases $v_{a}, v_{b}, v_{c}$ through the Park transform [7, 8].

The electric torque $\tau_{e}$ is given by

$$
\tau_{e}=\frac{3 p_{p}}{2}\left(\lambda_{m} i_{q}+\left(L_{d}-L_{q}\right) i_{d} i_{q}\right)
$$

where $p_{p}$ is the number of pole pairs. The mechanical dynamics equations are as follows:

$$
J \frac{d \omega_{r}}{d t}=\tau_{e}-\tau_{L}-B \omega_{r}, \quad \frac{d \theta}{d t}=\omega_{r}
$$

where $J$ is the total inertia referred to the respective motor shaft, $B$ is the viscous damping coefficient and $\tau_{L}$ is the load torque of the mechanical system. For constant flux operation when $i_{d}$ equals zero, the electrictorque $\tau_{e}=\frac{2}{3} \lambda_{m} i_{q}=K_{t} i_{q}$, where $K_{t}$ is the motor torque constant. Note that this torque equation for the PMSM resembles that of the regular DC machine and hence provides ease of control. For a uniform airgap surfaced-mounted PMSM motor, the $d$ - and $q$-axes inductances of the stator are equal, $L=L_{d}=L_{q}$. Thus the dynamic model of a surface-mounted PMSM can be described as follows: 


$$
\begin{aligned}
\dot{\theta} & =\omega_{r} \\
\dot{\omega}_{r} & =\frac{3 p_{p} \lambda_{m}}{2 J} i_{q}-\frac{B}{J} \omega_{r}-\frac{1}{J} \tau_{L} \\
\dot{i}_{q} & =-\frac{R_{s}}{L} i_{q}-\omega_{r} i_{d}-\frac{\lambda_{m}}{L} \omega_{r}+\frac{1}{L} u_{q} \\
\dot{i}_{d} & =-\frac{R_{s}}{L} i_{d}+\omega_{r} i_{q}+\frac{1}{L} u_{d}
\end{aligned}
$$

where $\theta$ is the rotor angle [rad], $J$ is the moment of inertia $\left[\mathrm{kg}-\mathrm{m}^{2}\right], \tau_{L}$ is the load torque [Nm], $p_{p}$ is the number of pole pairs, $B$ is the damping coefficient $[\mathrm{Nm} /(\mathrm{rad} / \mathrm{s})]$, and $u_{d}=v_{d}$ and $u_{q}=v_{q}$ are the $d$ and $q$ axes components of the voltages of the stator serving as the control variables $[\mathrm{V}]$.

The electrical rotor speed $\omega_{r}$ is related to the mechanical rotor speed $\omega_{m}$ by $\omega_{r}=p_{p} \omega_{m}$.

\section{Position Controller Design and Stability Analysis}

\subsection{Controller Design}

We define the position tracking error $z_{1}$ as $z_{1}=\theta-\theta_{d}$ where $\theta_{d}$ is the desired position. The states of the closed-loop system, $z_{c l}$ are defined as $z_{c l}=\left[z_{1}, z_{2}, z_{3}, z_{4}\right]^{T}$, where $z_{2}, z_{3}$ and $z_{4}$ are defined in the following theorem.

Theorem: Consider the dynamic model (9) of the PMSM, and $\left|z_{l}(0)\right|<k_{b}$. Suppose that the control law is given by

$$
\begin{aligned}
z_{1} & =\theta-\theta_{d} \\
\alpha_{1} & =-k_{1} z_{1}\left(k_{b}^{2}-z_{1}^{2}\right) \\
z_{2} & =\omega_{r}-\omega_{d} \\
\alpha_{2} & =\frac{2 J}{3 p_{p} \lambda_{m}}\left(-\frac{z_{1}}{k_{b}^{2}-z_{1}^{2}}-k_{2} z_{2}+\frac{B}{J} \omega+\frac{\tau_{L}}{J}+\dot{\omega}_{d}+\dot{\alpha}_{1}\right)-u_{q}^{d}, \\
z_{3} & =i_{q}-i_{q}^{d}-\alpha_{2} \\
z_{4} & =i_{d}-i_{d}^{d} \\
u_{q} & =L\left(-z_{2}-k_{3} z_{3}+\frac{R}{L} i_{q}+p \omega_{r} i_{d}+\frac{p \lambda_{m}}{L} \omega_{r}+\dot{i}_{q}^{d}+\dot{\alpha}_{2}\right) \\
u_{d} & =L\left(-k_{4} z_{4}+\frac{R}{L} i_{d}-p \omega_{r} i_{q}+i_{d}^{d}\right)
\end{aligned}
$$

where $k_{1}, k_{2}, k_{3}$ and $k_{4}$ are the positive controller gains, and $k_{b}$ is the tolerance of the position tracking error of the constraint, $\left|z_{0}\right|<k_{b}$. If $\left|z_{l}(0)\right|<k_{b}$, then $\left|z_{l}(t)\right|<k_{b}, \forall t>0$ and $z_{c l}(t)$ asymptotically converges to zero.

\subsection{Stability Analysis}

We use the beackstepping technique to prove the closed-loop stability of the proposed method using the barrier Lyapunov function.

Step 1: Let us define the barrier Lyapunov candidate function $V_{1}$ as follows:

$$
V_{1}=\frac{1}{2} \log \left(\frac{k_{b}^{2}}{k_{b}^{2}-z_{1}^{2}}\right)
$$

where $\log (\cdot)$ is the natural $\operatorname{logarithm}$ function. The derivative of $\mathrm{V} 1$ with respect to time is given by

$$
\dot{V}_{1}=\frac{z_{1} \dot{z}_{1}}{k_{b}^{2}-z_{1}^{2}}=\frac{z_{1}\left(z_{2}+\alpha_{1}\right)}{k_{b}^{2}-z_{1}^{2}} .
$$

Substituting $\alpha_{1}$ with that in (10) results in

$$
\dot{V}_{1}=-k_{1} z_{1}^{2}+\frac{z_{1} z_{2}}{k_{b}^{2}-z_{1}^{2}} \text {. }
$$


Step 2: Let us define $V_{2}$ as

$$
V_{2}=V_{1}+\frac{1}{2} z_{2}^{2}
$$

Then

$$
\dot{V}_{2}=\dot{V}_{1}+z_{2} \dot{z}_{2}=\dot{V}_{1}+z_{2}\left(\frac{3 p_{p} \lambda_{m}}{2 J}\left(z_{3}+i_{q}^{d}+\alpha_{2}\right)-\frac{B}{J} \omega_{r}-\frac{\tau_{L}}{J}-\dot{\omega}_{d}-\dot{\alpha}_{1}\right) .
$$

With $\alpha_{2}$, the time derivative of $V_{2}$ becomes

$$
\dot{V}_{2}=-k_{1} z_{1}^{2}-k_{2} z_{2}^{2}+z_{2} z_{3}
$$

Final step: We defined the overall Lyapunov candidate function $V_{b l f}$ as

$$
V_{b l f}=V_{2}+\frac{1}{2} z_{3}^{2}+\frac{1}{2} z_{4}^{2}
$$

We then obtain $\dot{V}_{3}$ as

$$
\begin{aligned}
\dot{V}_{b l f}= & \dot{V}_{2}+z_{3} \dot{z}_{3}+z_{4} \dot{z}_{4} \\
= & -k_{1} z_{1}^{2}-k_{2} z_{2}^{2}+z_{2} z_{3} \\
& +z_{3}\left(-\frac{R}{L} i_{q}-p \omega_{r} i_{d}-\frac{p_{p} \lambda_{m}}{L} \omega_{r}+\frac{1}{L} u_{q}-i_{q}^{d}-\dot{\alpha}_{2}\right)+z_{4}\left(-\frac{R}{L} i_{d}+p_{p} \omega_{r} i_{q}+\frac{1}{L} u_{d}-\dot{i}_{d}^{d}\right) .
\end{aligned}
$$

With the control input $u_{d}$ and $u_{q}, \dot{V}_{b l f}$ can be written as

$$
\dot{V}_{b l f}=-k_{1} z_{1}^{2}-k_{2} z_{2}^{2}-k_{3} z_{3}^{2}-k_{4} z_{4}^{2} \leq 0 .
$$

Thus, if $\left|z_{I}(0)\right|<k_{b}$, then $\left|z_{I}(t)\right|<k_{b}, \forall \mathrm{t}>0$ and $z_{c l}(t)$ asymptotically converges to zero.

\section{Conclusion}

We reviewed the dynamic modeling of the permanent magnet synchronous motor. The rotor angle position control method with error constraint is designed, and the closed-loop stability of the proposed method is studied using the Lyapunov theorem.

\section{Acknowledgments}

This research was supported by part of the Core Industry Technology Development Project of the Ministry of Trade, Industry and Energy, "Next Generation Manufacturing Robot for Smart Factory andWork- Space Sharing with Human.", Grant number 10063413.

\section{References}

[1] M. Krstic, I. Kanellakopoulos, and P. Kokotovic, "Nonlinear and adaptive control design," 1995, New York: Wiley.

[2] J. Zhoua, Y. Wang, "Real-time nonlinear adaptive backstepping speed control for a PM synchronous motor," Control Engineering Practice, vol. 13, pp. 1259-1269, 2005.

[3] K. P. Tee, S. S. Ge, and, E. H. Tay, "Barrier Lyapunov functions for the control of output constrained nonlinear systems," Automatica, vol. 45, no. 4, pp. 918-927, 2009. 
[4] K. P. Tee, S. S. Ge, and, E. H. Tay, "Output-feedback adaptive control of electrostatic microactuators," in Proceeding American Control Conference, pp. 4215-4220, 2009.

[5] B. V. E. How, S. S. Ge, and Y. S. Choo, "Control of coupled vessel, crane, cable, and payload dynamics for subsea installation operations," IEEE Transaction Control System Technology, vol. 19, pp. 208-220.

[6] M. Mikhov, "Mathematical Modeling and Dynamic Simulation of a Class of Drive Systems with Permanent Magnet Synchronous Motors," Applied and Computational Mechanics, vol. 3, pp. 331-338, 2009.

[7] P. Pillay, and R. Krishnan, "Modeling of permanent magnet motor drives," IEEE Transactions on Industrial Electronics, vol. 35, no. 4, pp. 537-541, 1988.

[8] P. C. Krause, Analysis of electric machinery, 2nd ed. New York: McGraw-Hill, 1995. 